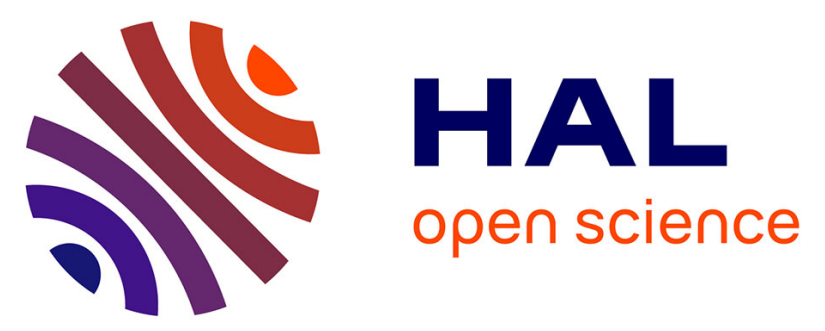

\title{
Kinetics of the coagulation cascade including the contact activation system: sensitivity analysis and model reduction
}

\author{
Rodrigo Méndez Rojano, Simon Mendez, Didier Lucor, Alexandre Ranc, \\ Muriel Giansily-Blaizot, Jean-François Schved, Franck Nicoud
}

\section{To cite this version:}

Rodrigo Méndez Rojano, Simon Mendez, Didier Lucor, Alexandre Ranc, Muriel Giansily-Blaizot, et al. Kinetics of the coagulation cascade including the contact activation system: sensitivity analysis and model reduction. Biomechanics and Modeling in Mechanobiology, 2019, 18 (4), pp.1139-1153. 10.1007/s10237-019-01134-4 . hal-02873826

\section{HAL Id: hal-02873826 \\ https://hal.umontpellier.fr/hal-02873826}

Submitted on 25 Nov 2020

HAL is a multi-disciplinary open access archive for the deposit and dissemination of scientific research documents, whether they are published or not. The documents may come from teaching and research institutions in France or abroad, or from public or private research centers.
L'archive ouverte pluridisciplinaire HAL, est destinée au dépôt et à la diffusion de documents scientifiques de niveau recherche, publiés ou non, émanant des établissements d'enseignement et de recherche français ou étrangers, des laboratoires publics ou privés. 


\title{
Kinetics of the coagulation cascade including the contact activation system:
}

\section{Sensitivity analysis and model reduction}

\author{
Rodrigo Méndez Rojano - Simon Mendez • Didier Lucor • Alexandre \\ Ranc - Muriel Giansily-Blaizot • Jean-François Schved · Frank Nicoud
}

Received: date / Accepted: date

\begin{abstract}
Thrombus formation is one of the main issues in the development of blood-contacting medical devices. This article focuses on the modeling of one aspect of thrombosis, the coagulation cascade, which is initiated by the contact activation at the device surface and forms thrombin. Models exist representing the coagulation cascade by a series of reactions, usually solved in quiescent plasma. However, large parameter uncertainty involved in the kinetic models can affect the predictive capabilities of this approach. In addition, the large number of reactions of the kinetic models prevents their use in the simulation of complex flow configurations encountered in medical devices. In the current work, both issues are addressed to improve the applicability and fidelity of kinetic models. A sensitivity analysis is performed by two different techniques to identify the most sensitive parameters of an existing detailed kinetic model of the coagulation cascade. The results are used to select the form of a novel reduced model of the coagulation cascade which relies on eight chemical reactors only. Then, once its parameters have been calibrated thanks to the Bayesian inference, this
\end{abstract}

Rodrigo Méndez Rojano · Simon Mendez · Franck Nicoud IMAG, Univ Montpellier, CNRS,

Montpellier, France E-mail: franck.nicoud@umontpellier.fr

Didier Lucor

LIMSI, CNRS, Université Paris-Saclay,

Orsay, France

Alexandre Ranc · Muriel Giansily-Blaizot · Jean-François Schved

Department of Haematology Biology,

CHU, Univ Montpellier,

Montpellier, France model shows good predictive capabilities for different initial conditions.

Keywords Coagulation Cascade Modeling · Sensitivity Analysis · Bayesian Inference · Model reduction

\section{Introduction}

The use of medical devices in contact with blood has been increasing over the last years (Sun et al. 2009; Alderazi et al. 2014; Indolfi et al. 2016; Bhogal et al. 2017; Kirklin et al. 2017). Such devices are used to treat cardiovascular or neurovascular disorders such as coronary artery diseases, heart valve diseases or aortic/brain aneurysms. One of the main problems of bloodcontacting devices is thrombus formation which can lead to device malfunction or thromboembolism (Mehra et al. 2014; Wilson and Cruden 2013). Blood clotting in devices is regulated by a series of intertwined biological processes, such as protein adsorption, platelet activity, complement system and coagulation reactions, as reviewed by Gorbet and Sefton (2004). These mechanisms appear due to the presence of the device whose artificial material lacks the endothelial properties of the vessel. At the material surface, contact activation of factor XII (zymogen of the coagulation cascade) takes place, initiating a cascade of enzymatic reactions (Yan et al. 2018) that produce thrombin, a key coagulation enzyme that activates platelets and forms fibrin. At the same time, platelet adhesion and activation can also occur at the device surface, as explained by Jaffer et al. (2015). In the last stage of the thrombus formation process, aggregated platelets and polymerized fibrin form a stable clot that may hinder the device performance. 
Computational fluid dynamics (CFD) has been used to study the flow on medical devices and evaluate the risk of thrombosis (Yoganathan et al. 2004; Dumont et al. 2007). A straightforward evaluation of thrombosis risk can be performed using specific flow patterns, such as high shear stress yielding to platelet activation (Alemu and Bluestein 2007; Shadden and Hendabadi 2013) or stasis which promotes coagulation (De Biasi et al. 2015). Approaches focusing only on flow properties do not allow to study the biochemical processes, which play a major role in thrombus formation (Fogelson and Neeves 2015). More exhaustive methods accounting for platelet activity, the coagulation cascade, clot growth and its interaction with the flow have been developed for thrombus triggered by vessel injury (Leiderman and Fogelson 2011; Yazdani et al. 2017). However, only small computational domains $x \sim 100 \mu \mathrm{m}$ can be considered due to the complexity of these models. Since a straightforward application of complicated models is not possible in the complex flow configurations linked to biomedical devices, reduced models accounting for platelet adhesion, activation (by chemical and mechanical pathways) and clot-flow interaction have been developed (Taylor et al. 2016; Wu et al. 2016). Nevertheless, thrombin produced by the coagulation cascade triggered by the contact system has not been considered in a model of thrombus formation in devices.

The contact activation system has already been considered in kinetic models of the coagulation cascade (Chatterjee et al. 2010; Zhu 2007). The kinetic descriptions have been used in CFD models to study thrombin formation triggered by the device wall in a typical flow configuration by Méndez Rojano et al. (2018). However, a straightforward coupling with platelet-based models is challenging due to the large number of reactions present in the kinetic models and the large physical time that must be computed in a thrombus growth process. Another issue is the large uncertainty in reaction rates due to non-standardized parameter characterization or variations among different subjects (Danforth et al. 2009). In addition, according to Link et al. (2018) the use of Michaelis-Menten kinetics is questionable when enzymes and zymogens participate to several reactions. In such cases, the use of mass action descriptions of complex formation and dissociation may be more adapted to avoid any assumption on the reactions. Overall, the uncertainty related to the kinetic models may lead to poor results in thrombin production as explained by Belyaev et al. (2018).
Parameter uncertainty and kinetic model reduction have been addressed for models of thrombus generation initiated by Tissue Factor exposure which is related to endothelial damage. Danforth et al. (2009) performed a sensitivity analysis on the kinetic model of Hockin et al. (2002) to evaluate the sensitivity of thrombin generation to the 44 reaction rates that are present in the model. The model sensitivity was assessed using the one at time (OAT) methodology with linear spaced variations between 10 to $1000 \%$ of the usual values of the reaction rates. The model outputs were evaluated at eight different instants which characterize the different phases of thrombin generation (initiation, amplification and propagation). Danforth and coworkers found that the model was especially sensitive to uncertainty in five parameters involved in the formation of the $T F=V I I a$ complex which takes place at the initial part of the coagulation cascade. The authors suggested that improving the accuracy of the reaction rate measurement can improve the predictive capabilities of the whole kinetic model. In a posterior work, Danforth et al. (2012) conducted a sensitivity analysis of the initial concentrations of the eight coagulation factors involved in the model of Hockin et al. (2002). They found that variations in prothrombin and TFPI accounted for $16 \%$ and $32 \%$ of the modifications of the model output respectively. Pairwise changes were also investigated by the authors, leading to stronger variations. The authors observed that the pair ATIII with TFPI had the largest impact on the results. Anand et al. (2008) performed a similar sensitivity analysis as Danforth et al. (2009) using the model of Naidu and Anand (2014). Their results showed that the generation of fibrin was most sensitive to the rate governing the production of thrombin.

Link et al. (2018) recently proposed a methodology consisting of a tailored sensitivity analysis approach using the screening technique of Morris (1991) combined with a more intensive global analysis technique. The sensitivity analysis was performed for the detailed model of Fogelson AL (2012). Three quantities of interest (QoI) related to thrombin were studied: the lag time, the maximum relative rate of generation, and the final concentration. The analysis of Link et al. (2018) considers four cases of parameter variations: variable initial concentrations levels of biochemical species, kinetic rate constants, biophysical and platelet attributes and the combined effects of varying the parameters all together. Their results show that the largest variations are observed when the combined effects of parameters variation yield to the largest effect on coagulation. Among the three studies considering only individual variations, modifications on the kinetic parameters had the largest 
effect on the production of thrombin.

In terms of model reduction, Wagenvoord et al. (2006) showed that a model with a small number of reactions can reproduce the thrombin generation curve if it includes the basic mechanism of thrombin formation: initiation, amplification and propagation. Papadopoulos et al. (2014) developed a minimal model for thrombin formation based on experimental data including these essential mechanisms. Reduced-order models allow computations in complex flow configurations. For instance, Ngoepe and Ventikos (2016) used the reduced model of Wagenvoord et al. (2006) to simulate thrombosis growth inside a cerebral aneurysm.

In the present work, a sensitivity analysis and a Bayesian inference method are used to reduce devicerelated kinetic models. First, the kinetic model of Chatterjee et al. (2010) initiated by the contact activation system is studied using a global screening technique that allows to identify the most sensitive parameters. Then, a reduced chemical model triggered by contact activation of factor $X I I$ is proposed. The optimal parameters of the reduced model are obtained using a data-driven Bayesian statistical framework to find the optimal parameters used in the reduced model.

\section{Methods}

\subsection{Plasma Samples and TGA}

Thrombin generation assay (TGA) was used to measure the evolution of thrombin concentration in time after coagulation was triggered by the contact activation system. Developed by Hemker et al. (2003), the Calibrated Automated Thrombogram (CAT) is a test mainly used in hemostasis research to study the hemostatic profile. This method is time-consuming and suitable only for a small number of samples when compared to routine clinical tests which assess the first traces of thrombin (after coagulation is triggered) about 12 seconds for Quick Time (QT) or 32 seconds for Activated Partial Thromboplastin Time (APTT) for a normal plasma. In contrast, TGA assesses thrombin generation until 60 minutes and allows a better characterization of the coagulation cascade throughout all its phases: initiation amplification and propagation. Any traces of thrombin will cleave to a specific substrate Z-Gly-Gly-Arg-AMC into a flurogenic form, detected by the thrombogram.

Samples: Each biological assays was performed with the Pooled Normal Plasma (PNPß) [Cryopep, Montpellier France] which consists of and industrial pooled citrated platelet poor plasmas (PPP) obtained from at least 20 healthy donors to avoid inter-individual physiological variations of clotting factor levels. Each donor has been pretested for QT, APTT, factor II, V, VII, VIII, IX, X, XI, XII levels, which all were between normal range. The PNP was diluted with prothrombin immuno-deficient plasma whose qualified activity was lower than 1\% [Siemens Healthcare, Erlangen, Germany] in order to obtain different final prothrombin concentrations. Silica was used to trigger the coagulation cascade, mixed with rabbit cephalin in STA-PPT A [Stago, Asnieres-sur-Seine, France].

Calibrated automated measurement of thrombin generation: Thrombin generation was determined in PPP using Fluoroscan Ascent (Flucakit, Thrombinoscope, Synapse BV, Maastrich, The Netherlands) according to the method described by Hemker et al. (2003). 80 $\mu \mathrm{L}$ of plasma were mixed with $20 \mu \mathrm{L}$ of STA-PPT A and $20 \mu \mathrm{L}$ of fluorescent reagent, FlucaKit. This reagent contains calcium chloride (necessary to trigger the coagulation cascade) and a mixture of fluorogenic substrate (Fluo-Substrate) and FluoBuffer. Thus, fluorescence intensity was detected at wavelengths of $390 \mathrm{~nm}$ (excitation filter) and $460 \mathrm{~nm}$ (emission filter), every 20 seconds. Each individual sample is analyzed with a thrombin calibrator as reference for a stable thrombin activity of approximately $600 \mathrm{nM}$. The calibrator enables the conversion of the fluorescence signal into thrombin concentration. The signal is treated to correct inner filtering effects, substrate consumption and abnormal plasma color. Analyses were conducted, on Immulon 2HB round-bottom 96-well plates (Stago Asnières-sur-Seine, France).

\subsection{Detailed coagulation model}

Sensitivity analysis is performed on the model introduced by Chatterjee et al. (2010). The model includes 37 reactions and 63 reaction rates and features:

- Initiation by the contact activation system considering factor XII auto-activation, reciprocal activation (by Kallikrein) and auto-hydrolysis (by factor $\left.X I I_{a}\right)$,

- Extrinsic and common pathways following the model of Hockin et al. (2002),

- Inhibition including Antithrombin (ATIII), Kallikrein inhibitor $K_{i n h}$ and $C_{1}$ inhibitor $C 1_{i n h}$.

The TGA experimental data generated in our laboratory was use as a test case for the model of Chatterjee et al. (2010) with some modifications related to 


\begin{tabular}{|c|c|c|c|c|}
\hline \# & Reaction & Forward $M^{-1} s^{-1}$ & Reverse $s^{-1}$ & Forward $s^{-1}$ \\
\hline 1 & $X a+V I I \rightarrow X a+V I I a$ & $k_{1}=1.3 \times 10^{7}$ & & \\
\hline 2 & $I I a+V I I \rightarrow I I a+V I I a$ & $k_{2}=2.3 \times 10^{4}$ & & \\
\hline 3 & $I I+X a \rightarrow I I a+X a$ & $k_{3}=7.5 \times 10^{3}$ & & \\
\hline 4 & $I I a+V I I I \rightarrow I I a+V I I I a$ & $k_{4}=2.0 \times 10^{7}$ & & \\
\hline 5 & $V I I I a+I X a \leftrightarrow I X a=V I I I a$ & $k_{5}=1.0 \times 10^{7}$ & $k_{6}=5.0 \times 10^{-3}$ & \\
\hline 6 & $\begin{array}{l}I X a=V I I I a+X \leftrightarrow I X a=V I I I a=X \rightarrow \\
I X a=V I I I a+X a\end{array}$ & $k_{7}=1.0 \times 10^{8}$ & $k_{8}=1.0 \times 10^{-3}$ & $k_{9}=8.2$ \\
\hline 7 & $V I I I a \leftrightarrow V I I I a_{1} \cdot L+V I I I a_{2}$ & & $k_{10}=6.0 \times 10^{-3}$ & $k_{11}=2.2 \times 10^{4}$ \\
\hline 8 & $\begin{array}{l}I X a=V I I I a=X \rightarrow V I I I a_{1} \cdot L+V I I I a_{2}+ \\
X+I X a\end{array}$ & & $k_{12}=1.0 \times 10^{-3}$ & \\
\hline 9 & $I X a=V I I I a \rightarrow V I I I a_{1} \cdot L+V I I I a_{2}+I X a$ & & $k_{13}=1.0 \times 10^{-3}$ & \\
\hline 10 & $I I a+V \rightarrow I I a+V a$ & $k_{14}=2.0 \times 10^{7}$ & & \\
\hline 11 & $X a+V a \leftrightarrow X a=V a$ & $k_{15}=4.0 \times 10^{8}$ & $k_{16}=0.2$ & \\
\hline 12 & $\begin{array}{l}X a=V a+I I \leftrightarrow X a=V a=I I \rightarrow X a= \\
V a+m I I a\end{array}$ & $k_{17}=1.0 \times 10^{8}$ & $k_{18}=103$ & $k_{19}=63.5$ \\
\hline 13 & $X a=V a+m I I a \rightarrow X a=V a+I I a$ & $k_{20}=1.5 \times 10^{7}$ & & \\
\hline 14 & $X a+T F P I \rightarrow X a=T F P I$ & $k_{21}=9.0 \times 10^{5}$ & $k_{22}=3.6 \times 10^{-4}$ & \\
\hline 15 & $X a+A T I I I \rightarrow X a=A T I I I$ & $k_{23}=1.5 \times 10^{3}$ & & \\
\hline 16 & $m I I a+A T I I I \rightarrow m I I a=A T I I I$ & $k_{24}=7.1 \times 10^{3}$ & & \\
\hline 17 & $I X a+A T I I I \rightarrow I X a=A T I I I$ & $k_{25}=4.9 \times 10^{2}$ & & \\
\hline 18 & $I I a+A T I I I \rightarrow I I a=A T I I I$ & $k_{26}=7.1 \times 10^{3}$ & & \\
\hline 19 & $B o c V P R M C A+I I a \rightarrow B o c V P R M C A=I I a$ & $k_{27}=1.0 \times 10^{8}$ & $k_{28}=6.1 \times 10^{3}$ & $k_{29}=53.8$ \\
\hline 20 & $X I I \rightarrow X I I a$ & $k_{30}=5.0 \times 10^{-3}$ & & \\
\hline 21 & $X I I a+X I I \leftrightarrow X I I a=X I I \rightarrow X I I a+X I I a$ & $k_{31}=1 \times 10^{8}$ & $k_{32}=750$ & $k_{33}=3.3 \times 10^{-2}$ \\
\hline 22 & $X I I a+P K \leftrightarrow X I I a=P K \rightarrow X I I a+K$ & $k_{34}=1 \times 10^{8}$ & $k_{35}=3.6 \times 10^{3}$ & $k_{36}=40$ \\
\hline 23 & $X I I+K \leftrightarrow X I I=K \rightarrow X I I a+K$ & $k_{37}=1 \times 10^{8}$ & $k_{38}=45.3$ & $k_{39}=5.7$ \\
\hline 24 & $P K+K \rightarrow K+K$ & $k_{40}=2.7 \times 10^{4}$ & & \\
\hline 25 & $K \rightarrow K i n h$ & & & $k_{41}=1.1 \times 10^{-2}$ \\
\hline 26 & $X I I a+C 1 i n h \rightarrow X I I a=C 1 i n h$ & $k_{42}=3.6 \times 10^{3}$ & & \\
\hline 27 & $X I I a+A T I I I \rightarrow X I I a=A T I I I$ & $k_{43}=21.6$ & & \\
\hline 28 & $X I+I I a \leftrightarrow X I=I I a \rightarrow X I a+I I a$ & $k_{44}=1 \times 10^{8}$ & $k_{45}=5$ & $k_{46}=1.3 \times 10^{-4}$ \\
\hline 29 & $X I I a+X I \leftrightarrow X I I a=X I \rightarrow X I I a+X I a$ & $k_{47}=1.0 \times 10^{9}$ & $k_{48}=200$ & $k_{49}=5.7 \times 10^{-3}$ \\
\hline 30 & $X I a+X I \rightarrow X I a+X I a$ & $k_{50}=3.19 \times 10^{6}$ & & \\
\hline 31 & $X I a+A T I I I \rightarrow X I a=A T I I I$ & $k_{51}=3.2 \times 10^{2}$ & & \\
\hline 32 & $X I a+C 1 i n h \rightarrow X I a=C 1 i n h$ & $k_{52}=1.8 \times 10^{3}$ & & \\
\hline 33 & $X I a+\alpha_{1}-A T \rightarrow X I a=\alpha_{1}-A T$ & $k_{53}=1.0 \times 10^{2}$ & & \\
\hline 34 & $X I a+\alpha_{2}-A P I \rightarrow X I a=\alpha_{2}-A P$ & $k_{54}=4.3 \times 10^{3}$ & & \\
\hline 35 & $X I a+I X \leftrightarrow X I a=I X \rightarrow X I a+I X a$ & $k_{55}=1.0 \times 10^{8}$ & $k_{56}=41.0$ & $k_{57}=7.7$ \\
\hline 36 & $I X a+X \leftrightarrow I X a=X \rightarrow I X a+X a$ & $k_{58}=1.0 \times 10^{8}$ & $k_{59}=0.64$ & $k_{60}=7.0 \times 10^{-4}$ \\
\hline 37 & $X a+V I I I \leftrightarrow X a=V I I I \rightarrow X a+V I I I a$ & $k_{61}=1.0 \times 10^{8}$ & $k_{62}=2.1$ & $k_{63}=0.023$ \\
\hline
\end{tabular}

Table 1 Kinetic parameters and coagulation reactions used in the simulations. The model of Chatterjee et al. (2010) was adapted to the experimental conditions detailed in Section 2.1 .

our experimental setup. The inhibition by corn trypsin inhibitor was suppressed since it was not used. The initial trigger reaction activation of factor XII (reaction 20) was modified, the original value of Chatterjee et al. (2010) being fitted to their specific experimental framework. Fibrin related reactions were not included in the model to reduce the computational cost of the model when performing the sensitivity analysis. The reactions related to factor $X$ and factor $I X$ activation by factor $V I I_{a}$ were deleted because those reactions referred to the non-physiological recombinant factor $V I I_{a}$ (Komiyama et al. 1990) (these reactions are numbered 48 and 49 in Chatterjee et al. (2010)). The prediction of the resulting model is plotted in Fig. 1 in dash-dotted line. It was observed that the model systematically overestimates the lag time. In order to improve the baseline comparison between experiments and model predictions, the kinetic rates $k_{47}$ and $k_{49}$ were augmented by a factor of 10 with respect to the values reported by Chatterjee et al. (2010). The result is plotted in Fig. 1 as solid line and compares well with the experimental data. All the reactions and the baseline values of the reaction rates are reported in Table 1 . Note that these modifications drastically improve the numerical-experimental comparison in the thrombin generation test cases considered in this study.

A system of ordinary differential equations (ODE) can be written from the biochemical reactions of the kinetic model (Table 1). The ODE system writes:

$$
\frac{d C_{i}}{d t}=R_{i}
$$

where $C_{i}$ is the concentration of species $i$ and $R_{i}$ is the source term which is obtained by applying the law of mass action to the coagulation reactions of Table 1 . As an example the source term of Kininogen $K$ is devel- 
oped as:

$$
\begin{aligned}
\frac{d C_{K}}{d t}= & -k_{41} C_{K}-k_{37} C_{X I I} C_{K}+\left(k_{38}+k_{39}\right) C_{X I I=K} \\
& +2 k_{40} C_{P K} C_{K}+k_{36} C_{X I I_{a}=P K}
\end{aligned}
$$

The solution of the system is computed using an integration Runge-Kutta scheme of the 4th order. This numerical scheme was implemented in the in-house CFD solver YALES2BIO ${ }^{1}$. YALES2BIO has already been used in coagulation problems by Méndez Rojano et al. (2018) and other cardiovascular flows in micro (Sigüenza et al. 2017; Lanotte et al. 2016) and macro (Chnafa et al. 2016; Zmijanovic et al. 2017; Sigüenza et al. 2018) scale applications.

Table 2 shows the initial concentrations used in the numerical simulations which correspond to the physiological concentrations of coagulation factors after sample preparation.

In addition to the concentrations of species, which are patient-specific, the coagulation model is governed by 63 reaction rates (see Table 1 ). The reaction rates are derived from reactions observed under experimental conditions of saturating phospholipid concentration. Most of the kinetics models are build upon reaction rate values reported in literature (Jones and Mann 1994; Zhu 2007; Zarnitsina et al. 1996). Additional fitting is in general required due to non-physiological conditions in which some parameters are derived or extensively modified proteins which are used to obtain the parameter value (Hockin et al. 2002). For this reason, reported values in different kinetic models sometimes present large variation. For instance, the reaction rate of factor $X I$ activation can be found in literature with a difference up to 5 orders of magnitude as highlighted by Belyaev et al. (2018).

Figure 1 shows the numerical and experimental data. The numerical set up here presented was used as a nominal reference for the sensitivity analysis. A reasonable agreement between the experimental TGA and the kinetic model of Chatterjee et al. (2010) was obtained.

\subsection{Global Sensitivity Analysis by Morris screening}

The objective of the present sensitivity analysis is not to precisely quantify the influence of the kinetic parameters on coagulation as in Danforth et al. (2009) or Link et al. (2018), but rather to identify the most influencing

\footnotetext{
1 http://www.math.univ-montp2.fr/ yales2bio/
}

\begin{tabular}{cc}
\hline Factor & Initial Condition [nM] \\
\hline$V I I$ & 6.67 \\
$V I I a$ & 0.667 \\
$X$ & 106.67 \\
$I X$ & 6.0 \\
$I I$ & 933.0 \\
$V I I I$ & 0.4667 \\
$V$ & 13.33 \\
$T F P I$ & 1.667 \\
$A T I I I$ & 2267.0 \\
$X I I$ & 226.7 \\
$P K$ & 300.0 \\
$C 1_{\text {inh }}$ & 1667.0 \\
XI & 206.7 \\
$\alpha_{1}-A T$ & 30000.0 \\
$\alpha_{2}-A P$ & 667.0 \\
BocVPRMCA & 6670.0 \\
\hline
\end{tabular}

Table 2 Factors concentrations based on TGA (concentrations are calculated after sample mixing with fluorescent reagent and using the physiological values reported by Chatterjee et al. (2010)) used as initial conditions in the numerical simulations.

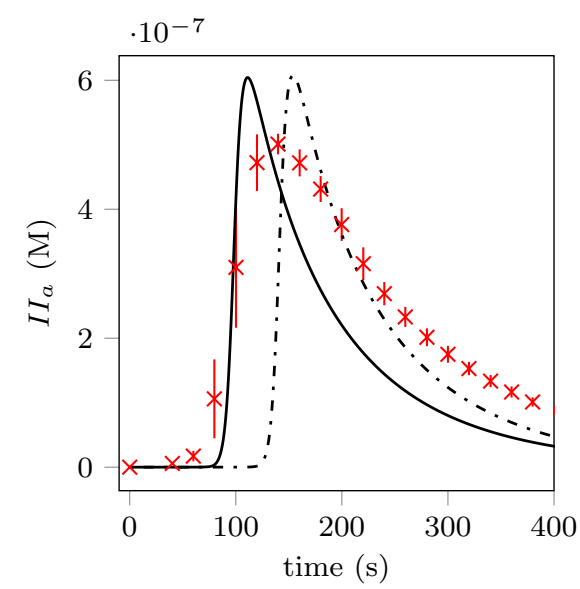

Fig. 1 Experimental results of thrombin generation assay $(\times)$ and numerical simulation ( - ) using the kinetic scheme of Table 1 with the initial conditions of Table 2. The numerical result without modifying $k_{47}$ and $k_{49}$ is also shown $(--\cdot)$.

kinetic rates. This is a necessary step in order to gain more insight into the model and use this knowledge to build up the reduced kinetic model of Section 3.2. The screening method of Morris (1991) allows a fast exploration of a model through the discretization of the input parameters. This type of method is well suited to models with many parameters and a good compromise between accuracy and efficacy has been reported (Iooss and Lemaître 2015). The Morris method was also recently used by Link et al. (2018), who highlighted its ability to rank the most sensitive parameters of a complex thrombosis model.

The general idea of the Morris analysis is to calculate a number of elementary effects for each input parameters and to compute basic statistics to identify the most sensitive parameters. In order to compute the elementary effects a discrete grid is built on the input variables and explored with an efficient sampling technique. In the 
current work, the python module SALIB ${ }^{2}$ that follows the optimized version of the Morris method by Campolongo et al. (2007) was used. We considered only the case of variations on the kinetic rates as they produced the largest change in the production of thrombin as observed in Link et al. (2018). The practical steps that were performed to conduct the analysis using the model of Chatterjee et al. (2010) are the following:

1. Choice of discrete grid and sampling: a discrete grid on the $p=63$ input variables is built with $l$ probability levels uniformly distributed between $[0,1]$. In this analysis 10 levels of discretization were used. Inside the input domain a number of trajectories $r$ has to be determined in order to cover the input parameters space in an efficient manner. In the original method of Morris (1991), the trajectories starting point is chosen randomly, then consecutive one-at-a-time increments are performed in the discrete grid with a random direction. To perform an optimal coverage of the input space, the optimized strategy of Campolongo et al. (2007) was preferred in this analysis. In the optimal strategy, the dispersion of the starting points is maximized through the generation of several Morris trajectories (in this case $\left.r_{\max }=500\right)$. Then the most spread optimal trajectories are kept, for this case $r=10$. The total number of experiments is then defined by $M=r(p+1)$ thus, for the current model, $M=640$ TGA simulations were required.

2. Mapping of parameters to actual distribution: once the sampling is performed a Design of Experiment (DoE) matrix with parameters between 0 and 1 is obtained. A quantile function is applied to transform the parameters to their actual values. This is a common practice in several screening strategies Saltelli et al. (2004). In our analysis, a loguniform probability distribution is used for all the input parameters. This choice was motivated by the large parameter variations reported in the literature (Hemker et al. 2012). In addition, the actual distribution for each reaction rate is not available in literature. The quantile function used writes

$Q\left(z_{i}\right)=c_{\text {inf }, i}\left(\frac{c_{\text {sup }, i}}{c_{\text {inf }, i}}\right)^{z_{i}}$

where $\left[c_{\text {inf }, i} ; c_{\text {sup }, i}\right]$ is the distribution support and $z_{i}$ is the sampled value of the $i$ parameter (between $[0,1])$. To guarantee that the mean $\mu_{i}$ of the sampled parameters coincides with the nominal input value $k_{i}$, the superior and the inferior support values must be estimated. To do so, the support ratio $\beta=\frac{c_{\text {sup }, i}}{c_{\text {inf }, i}}$

2 http://salib.readthedocs.io/en/latest/api.html is introduced in the log-uniform mean expression $\mu_{i}=\left(c_{\text {sup }, i}-c_{\text {inf }, i}\right) /\left(\log \left(c_{\text {sup }, i}\right)-\log \left(c_{\text {inf }, i}\right)\right)$. Imposing $\mu_{i}=k_{i}$ then leads to $c_{i n f, i}=\frac{k_{i} \log (\beta)}{\beta-1}$ and $c_{\text {sup }, i}=\beta c_{\text {inf }, i}$. In the current analysis, $\beta$ was set equal to 100 which translates in parameters variation of about $4.5 \%-450 \%$ of each parameter nominal values. Two trajectories in the $\left(k_{3}, k_{7}\right)$ plane are displayed in Figure 2 to illustrate the method.

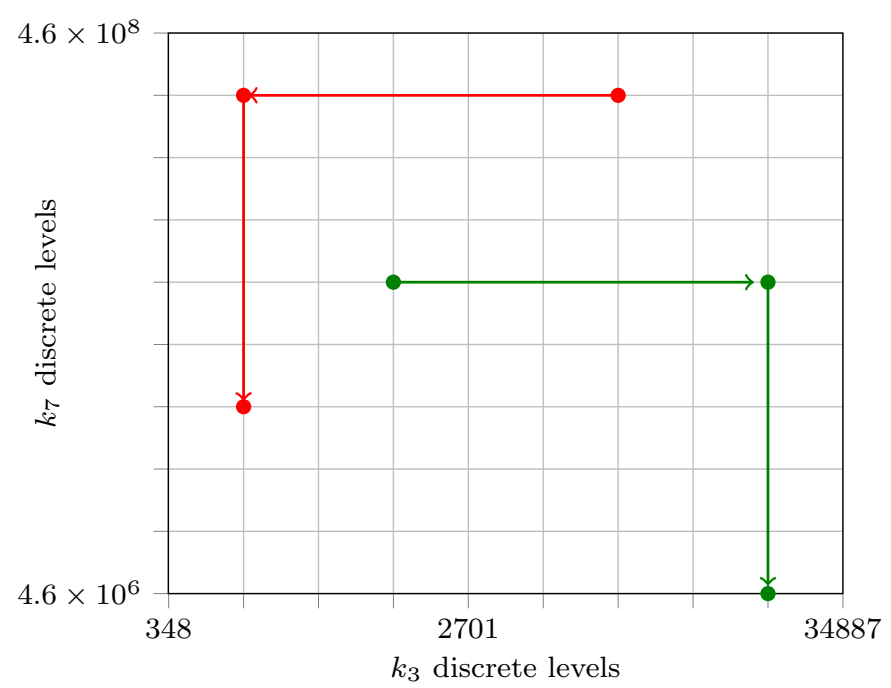

Fig. $22 \mathrm{D}$ parameter grid, the levels are mapped using the log uniform distribution. Two trajectories are shown as an ilustration of the OAT random advancement.

3. Solution with YALES2BIO and Quantities of Interest (QoI) post-processing: The DoE simulations were performed using YALES2BIO with a fixed time step of $\Delta t=1 \times 10^{-4} \mathrm{~s}$. Post-processing included the extraction of the QoI from the thrombin formation curve. The QoI used in the sensibility analysis are shown in Fig. 3. The five QoI are:

(a) $t_{\left(I I_{a}=10.0 n M\right)} \equiv t_{\text {lag }}$ : the instant at which the thrombin concentration reaches $10 \mathrm{nM}$,

(b) the maximum value of thrombin concentration $\max \left(I I_{a}\right)$

(c) the ascending slope $m_{1}$ defined from the lag point $\left(t_{l a g}, I I_{a}=10.0 n M\right)$ and the maximum peak point $\left(t_{\max }, \max \left(I I_{a}\right)\right)$,

(d) the descending slope $m_{2}$ defined with the maximum peak point $\left(t_{\max }, \max \left(I I_{a}\right)\right)$ and the descending point at $\left(t_{d}, I I_{a}=10 \mathrm{nM}\right)$ where $t_{d}$ is the instant at which the concentration of thrombin falls bellow $10 \mathrm{nM}$,

(e) endogenous thrombin potential (ETP) which stands for the area under the curve representing the total thrombin produced. 
4. Elementary effects and sensitivity indices: the OAT incremental ratios allow to compute elementary effects of each parameter following Campolongo et al. (2007):

$E_{j}^{i}=\frac{\left.f\left(X_{j}^{i}+\delta_{i}\right)-f\left(X_{j}^{i}\right)\right)}{\delta_{i}}$

where $X$ is the input parameter, $j$ is the index of the input parameter, $i$ stands for the repetition, $\delta_{i}$ is the variation value and the function $f$ represents any of the five QoI from the thrombin generation curve. Two sensitivity coefficients are calculated (Campolongo et al. 2007):

$-\mu_{j}^{*}=\frac{1}{r} \sum_{i=1}^{r}\left|E_{j}^{i}\right|$ is the mean of the absolute value of the elementary effects

$-\sigma_{j}=\sqrt{\frac{1}{r} \sum_{i=1}^{r}\left(E_{j}^{i}-\frac{1}{r} \sum_{i=1}^{r} E_{j}^{i}\right)^{2}}$ is the standard deviation of the elementary effects.

From the sensitivity indices distribution a quick identification of three different groups of parameters can be done depending on their impact on the QoI:

- parameters with little or no effect (low $\mu_{j}^{*}$ and $\left.\sigma_{j}\right)$

- parameters with a strong linear dependency or an additive effect (large $\mu_{j}^{*}$ ),

- parameters with non-linear and/or interaction effects (large $\sigma_{j}$ ),

With this classification the most sensitive parameters can be identified. In some cases, the sensitivity analysis can identify predominant reactions and contribute to reduce the complexity of the models, as discussed in Section 4 .

2.4 A reduced model of coagulation initiated by contact activation

Wagenvoord et al. (2006) proposed a minimal reaction mechanism of thrombin formation triggered by factor $V I I_{a}$. The reduced model included reactions of the extrinsic and common pathways and can thus be applied in cases were $T F$ is the initial mechanism. In this model, 14 parameters including 9 kinetic constants and 5 initial concentrations of coagulation factors must be optimized in order to correctly reproduce experimental trends of thrombin formation. It is worth mentioning that in reduced kinetic models, the species maybe fictive species and the optimal initial concentrations of factors are not representative of the actual concentrations in blood plasma. However, these models have been successful to reproduce thrombin formation triggered by the extrinsic pathway. In the current work a reduced model for

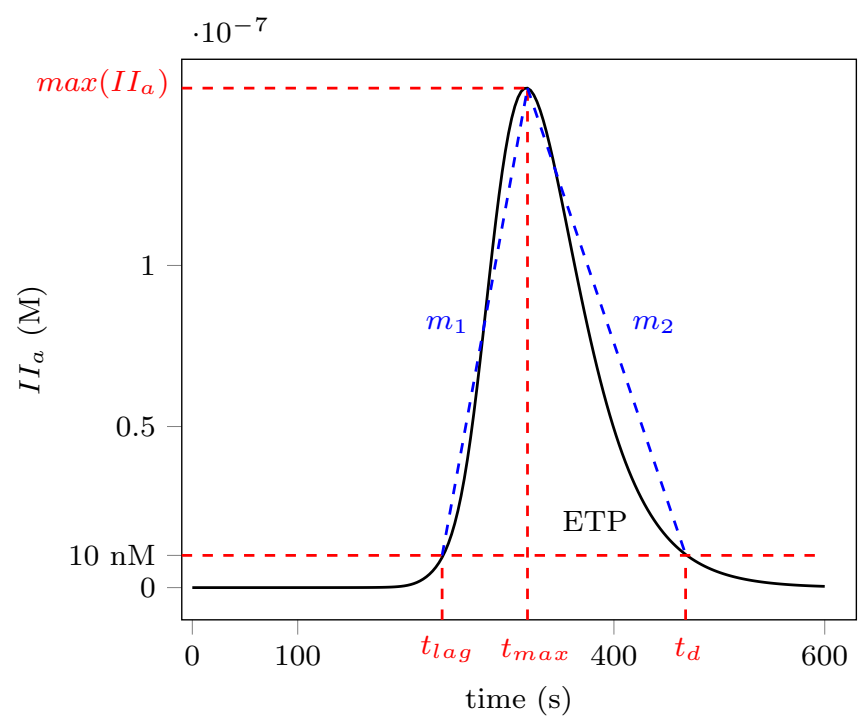

Fig. 3 Variables used in the sensibility analysis: $t_{l a g}$, $\max \left(I I_{a}\right), m_{1}, m_{2}, \mathrm{ETP}$.

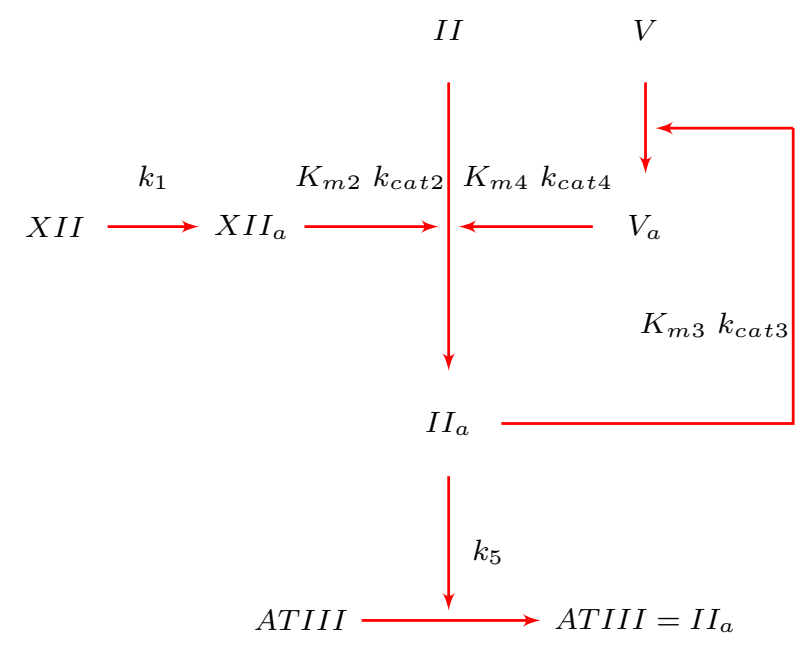

Fig. 4 Reduced model for thrombin generation triggered by the contact activation system. The notation $K_{m i}, k_{c a t i}$ is used for reaction following MichaelisMenten kinetics while $k_{i}$ are first order reactions.

thrombin production initiated by contact activation is proposed. The minimal reaction mechanisms consist of 5 reactions that are triggered by activation of factor $X I I$, the reactions are listed on Table. 3. The model includes 8 reaction rates and 8 chemical species. The amplification and propagation phases are performed by the auto-activation loop through the activation of factor $V_{a}$, this mechanism also appearing in the model of Wagenvoord et al. (2006). Finally, inhibition of thrombin is due to the activity of ATIII. Figure 4 shows a schematic of the model with the corresponding kinetic parameters. The kinetic model $\dot{\boldsymbol{C}}=\mathcal{M}(\boldsymbol{C}, t)$ can be 


\begin{tabular}{|c|c|c|c|c|}
\hline$\#$ & Reaction & $K_{m} \mathrm{M}$ & $k_{\text {cat }} \mathrm{s}^{-1}$ & $k \mathrm{~s}^{-1}$ \\
\hline 1 & $X I I \rightarrow X I I_{a}$ & & & $k_{1}$ \\
\hline 2 & $X I I_{a}+I I \rightarrow I I_{a}+X I I_{a}$ & $K_{m 2}$ & $k_{c a t 2}$ & \\
\hline 3 & $I I_{a}+V \rightarrow I I_{a}+V_{a}$ & $K_{m 3}$ & $k_{\text {cat } 3}$ & \\
\hline 4 & $V_{a}+I I \rightarrow V_{a}+I I_{a}$ & $K_{m 4}$ & $k_{\text {cat } 4}$ & \\
\hline 5 & $I I_{a}+A T I I I \rightarrow I I_{a}=A T I I I$ & & & $k_{5}$ \\
\hline
\end{tabular}

Table 3 Reduced kinetic model for the coagulation cascade showed in Fig. 4. Reactions 1 and 5 are first order reaction, reactions 2, 3 and 4 follow MichaelisMenten kinetics.

represented by a system of ordinary equations that can be solved using a classical numerical scheme. The ODE system writes:

$\left[\begin{array}{c}\dot{C}_{X I I} \\ \dot{C}_{X I I a} \\ \dot{C}_{I I} \\ \dot{C}_{I I a} \\ \dot{C}_{V} \\ \dot{C}_{V a} \\ \dot{C}_{A T I I I} \\ \dot{C}_{A T I I I=I I_{a}}\end{array}\right]=\left[\begin{array}{c}k_{1} C_{X I I} \\ \frac{k_{c a t 4} C_{V_{a}} C_{I I}}{K_{m 4}+C_{I I}}-\frac{k_{c a t 2} C_{X I I_{a}} C_{I I}}{K_{m 2}+C_{I I}}-k_{5} C_{I I_{a}} C_{A T I I I} \\ \frac{k_{c a t 2} C_{X I I_{a}} C_{I I}}{K_{m 2}+C_{I I}}+\frac{k_{c a t 4} C_{V_{a}} C_{I I}}{K_{m 4}+C_{I I}}-k_{5} C_{I I_{a}} C_{A T I I I} \\ -\frac{k_{c a t 3} C_{I I_{a}} C_{V}}{K_{m 3}+C_{V}} \\ \frac{k_{c a t 3} C_{I I_{a}} C_{V}}{K_{m 3}+C_{V}} \\ -k_{5} C_{I I_{a}} C_{A T I I I} \\ k_{5} C_{I I_{a}} C_{A T I I I}\end{array}\right]$

Parameter values should be optimized to compute thrombin formation with a reasonable agreement with reference data. Here we use the experimental data generated by TGA triggered by contact activation.

\subsection{Bayesian parameter inference}

The proposed reduced-order coagulation model depends on a set of parameters which need to be calibrated. The idea behind this parametric calibration is to adjust the unknown parameters in order to lower the discrepancy between the numerical prediction and some indirect and imperfect (experimental) observations of the system. This is a computational burden because the model is nonlinear and brings in unknown relatively high-dimensional parameter spaces. Model inversion in the presence of measurement errors must typically take advantage of some type of regularization (e.g., Tikhonov regularization) in order to recover the existence and uniqueness of solutions or a robust optimization method (Ashyraliyev et al. 2009). A potentially more natural setting is the Bayesian statistics. The Bayesian statistical framework can be explained as a systematic use of probability dedicated to decisionmaking when using a model with uncertain parameters.
It is advantageous because it allows the specification of a prior distribution which expresses probabilistically what is known about the parameters before observing the data. In this study, a Bayesian inference technique is used to obtain the optimal parameters involved in the model that allow to capture the thrombin formation curve. The parameters to be inferred are the 8 reactions rate and the initial concentrations of species $X I I, V$ and $A T I I I$, i.e. a total of 11 quantities.

This approach is used in inverse problems and relies on posterior sampling techniques. Thus, a prior distribution of the parameters to be calibrated is necessary, so that the estimation process delivers a probabilistic characterization of the parameters. In this work, a MarkovChain Monte-Carlo algorithm is used considering the input parameters as random quantities that are sampled according to the posterior distribution.

A significant amount of literature exists on the Bayesian inference method. In the following, the work of Xiu (2010); Birolleau et al. (2014); Andrieu et al. (2003) is referenced to recall the main steps of the parameter inference. In the Bayesian approach, a vector of unknown parameters is considered

$$
\begin{aligned}
& \boldsymbol{k}=\left(k_{1}, K_{m 2}, k_{c a t 2}, K_{m 3}, k_{c a t 3}, K_{m 4}, k_{c a t 4}, k_{5},\right. \\
& \left.X I I_{\text {init }}, V_{\text {init }}, A T I I I_{\text {init }}\right) .
\end{aligned}
$$

The parameters are treated as random variables with a prior density distribution $\pi_{\text {prior }}(\boldsymbol{k})$. In the current inference procedure, independent log-uniform distributions were assumed as prior distributions for all the parameters. Observations of the QoI are necessary to perform the inference process. In this application, the observed variables $\boldsymbol{d} \in \mathbb{R}^{n_{\boldsymbol{d}}}$ are the experimental measurements of the thrombin generation assay or the numerical results of the detailed kinetic model at $n_{t}$ discrete time instants $C_{I I_{a}}\left(n_{t}\right)$.

The Bayesian formula, involving conditional probabilities, can be applied as in Birolleau et al. (2014) to obtain the parameters posterior distribution considering the reduced model, the prior parameters distribu- 
tion and the experimental observations

$\pi_{\text {post }}(\boldsymbol{k} \mid \boldsymbol{d}) \propto \pi_{\ell}(\boldsymbol{d} \mid \boldsymbol{k}) \pi_{\text {prior }}(\boldsymbol{k})$,

where $\pi_{\ell}(\boldsymbol{d} \mid \boldsymbol{k})$ is the likelihood function that combines the experimental data with the forward model and $\pi_{\text {post }}(\boldsymbol{k} \mid \boldsymbol{d})$ the desired posterior density of the parameters. The initial concentration of prothrombin is not inferred, thus the physiological condition of pool plasma is used in the reduced kinetic model, $I I=933.0 \mathrm{nM}$. Following Xiu (2010) assuming additive measurement noise $\varepsilon$ and mutually independent random variables, one can write:

$\boldsymbol{d}=\mathcal{G}(\boldsymbol{C})+\boldsymbol{\varepsilon}=\mathcal{G}(\mathcal{M}(\boldsymbol{k}, t))+\boldsymbol{\varepsilon}$,

where $\mathcal{M}: \mathbb{R}^{n_{k}} \times \mathbb{R}^{n_{t}} \rightarrow \mathbb{R}^{n_{C} \times n_{t}}$ is the deterministic forward model, $\mathcal{G}: \mathbb{R}^{n_{\boldsymbol{C}} \times n_{t}} \rightarrow \mathbb{R}^{n_{\boldsymbol{d}}}$ is an observation operator that relates the model solution $\boldsymbol{C}$ with the reference discrete concentration of thrombin $\boldsymbol{d}$ and $\pi_{\varepsilon}$ is the prescribed noise distribution of $\varepsilon$. The observation operator $\mathcal{G}$ can be any operation that transforms the model outputs into the QoI used in the inference process. In the current case the operator $\mathcal{G}$ only selects the thrombin concentration $C_{I I_{a}}$ from the output concentration vector $\boldsymbol{C}$ of the forward model $\mathcal{M}$. More specifically, in our case, the likelihood function can be expressed as follows:

$\pi_{\ell}(\boldsymbol{d} \mid \boldsymbol{k})=\prod_{i=1}^{n_{d}} \varepsilon\left(d_{i}-\mathcal{G}_{i}(\mathcal{M}(\boldsymbol{k}, t))\right)$.

where the index $i$ is used to refer to the experimental and numerical observations at the same $n_{d}$ time instants. The likelihood function contains a stochastic source term that must encompass the response of the deterministic kinetic model over the support of $\pi_{\text {post }}(\boldsymbol{k} \mid \boldsymbol{d})$. Since the posterior distribution does not have in this case an analytic closed expression, posterior moments, expectations or maximum a posteriori values must be estimated via sampling methods such as Markov chain Monte Carlo (MCMC) as explained in Andrieu et al. (2003). The posterior sampling of the parameters is handled thanks to a standard Metropolis-Hastings scheme (Hastings et al. 2017). In our case, it requires many model realizations (about twenty thousand), which is not penalizing as the reduced-order model is not computationally demanding.

\section{Results}

3.1 Sensitivity Analysis of the full coagulation model

The first aspect of interest is the robustness of the detailed coagulation model of Chatterjee et al. (2010) ini- tiated by contact activation. Figure 5 shows the results of the sensitivity analysis using the screening Morris method for each quantity of interest. The basic statistics of the elementary effects $\sigma_{j}$ and $\mu_{j}^{*}$ are computed using the thrombin concentration of the 640 simulations. An arbitrary threshold of $30 \%$ of the largest value of both sensitivity indices is used to keep apart the sensitive parameters.

The important parameters identified for the time lag $t_{l a g}$ (Fig. 5a) belong to the intrinsic pathway; activation of factors $I X$ (reaction 35) and $X I$ (reaction $29)$ have large impacts on $t_{\text {lag }}$. The parameters relevant to $\max \left(I I_{a}\right)$ are reactions rates involved in the activation of factor $I I$ (Fig. $5 \mathrm{~b}$ ). In addition, thrombin inhibition due to ATIII presents a large influence on the maximum value of thrombin. The ascending slope $m 1$ (Fig. $5 \mathrm{c}$ ) shows a large influence to reaction rates involved in reaction 12 which is formation of $m I I_{a}$ by the Prothrombinase complex. Furthermore, reaction rates involved in the intrinsic pathway, activation of factors, $X I_{a}, I X, X$ and $V I I I$, showed to be important for $m_{1}$ for instance. The descending slope of thrombin evolution $m_{2}$ (Fig. $5 \mathrm{~d}$ ) is mostly influenced by the thrombin inhibition activity of $A T I I I$ and by the production of $m I I_{A}$ and $I I_{a}$ in reactions 12 and 13 in which kinetic rates 18 and 20 participate.

Finally, the results on ETP (Fig. 5e) show that the most influential parameters are involved in the inhibition activity of ATIII by means of complex formation with $I I_{a}$ and $m I I_{a}$. Reaction rate $k_{20}$ which is involved in the conversion of $m I I_{a}$ into $I I_{a}$ is also relevant to ETP. The histogram shows that the conversion of $m I I_{a}$ into $I I_{a}$ determined by $k_{20}$ is relevant to all the QoI. In a similar way, inhibition activity by $A T I I I$ due to parameter $k_{26}$ is important for 4 QoI, excluding only $t_{\text {lag }}$. Six reaction rates are sensitive to at least three QoI, the majority of these parameters are involved directly in the production of $I I_{a}$ with the exclusion of parameter $k_{60}$ which is involved in the activation of factor $X$ by $I X_{a}$.

The parameters identified by the sensitivity analysis are consistent with the nature of the QoI. It is important to note that these results were verified using a very different strategy for global sensitivity analysis. In this case, influential parameters were identified thanks to their first-order Sobol' indices (Sobol 2001). Sobol' indices are a variance-based diagnostic method of global sensitivity analysis that can identify the parameters that cause large variations on the output of a given model. 
a)

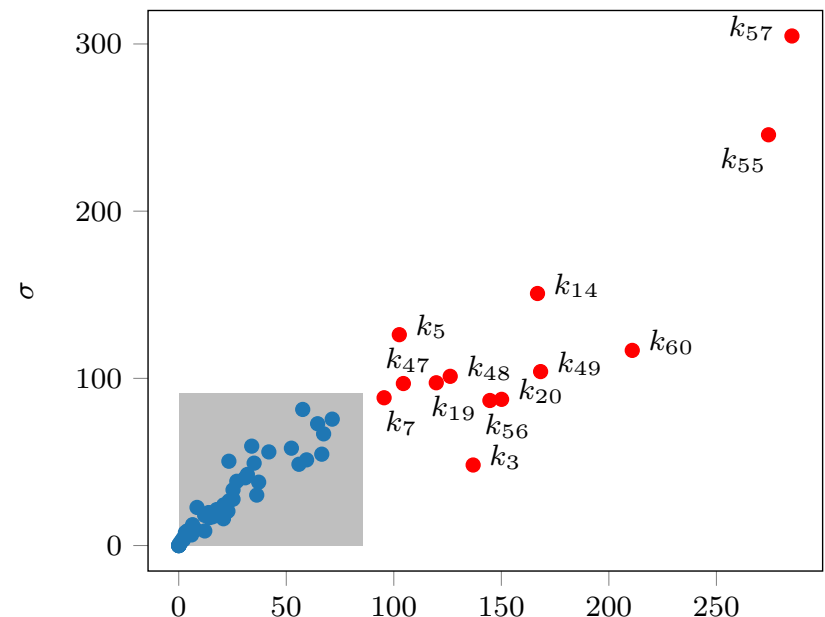

c)

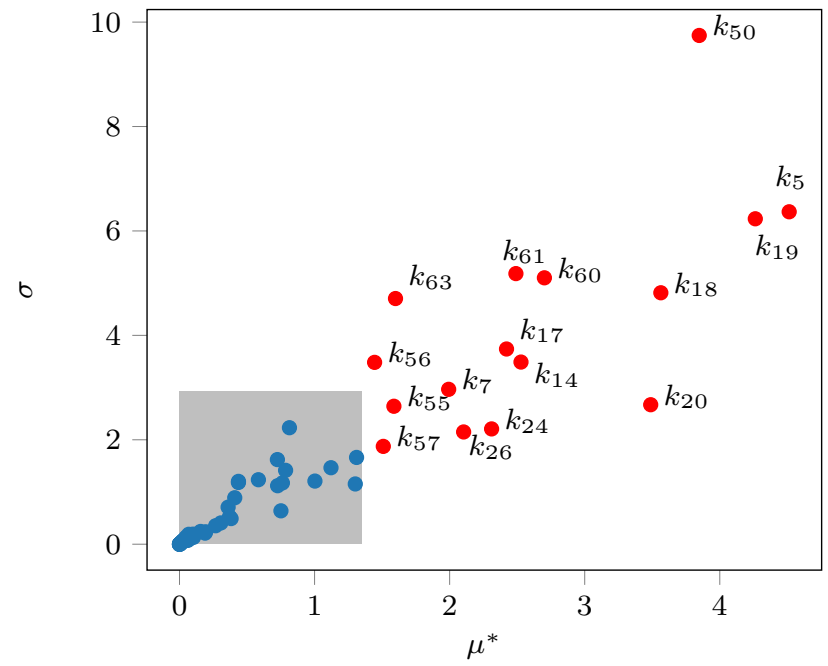

e)

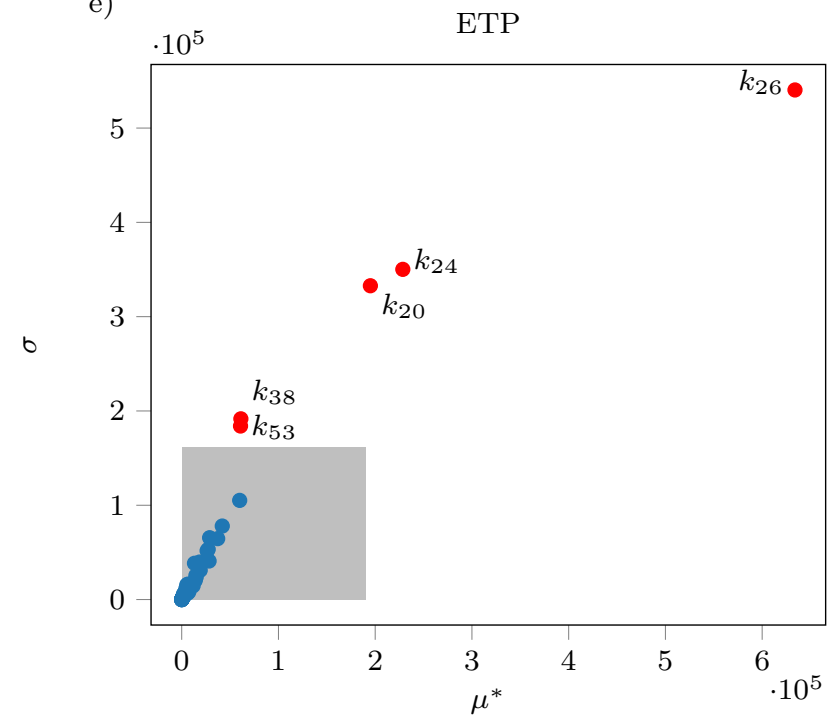

b)

$\max \left(I I_{a}\right)$

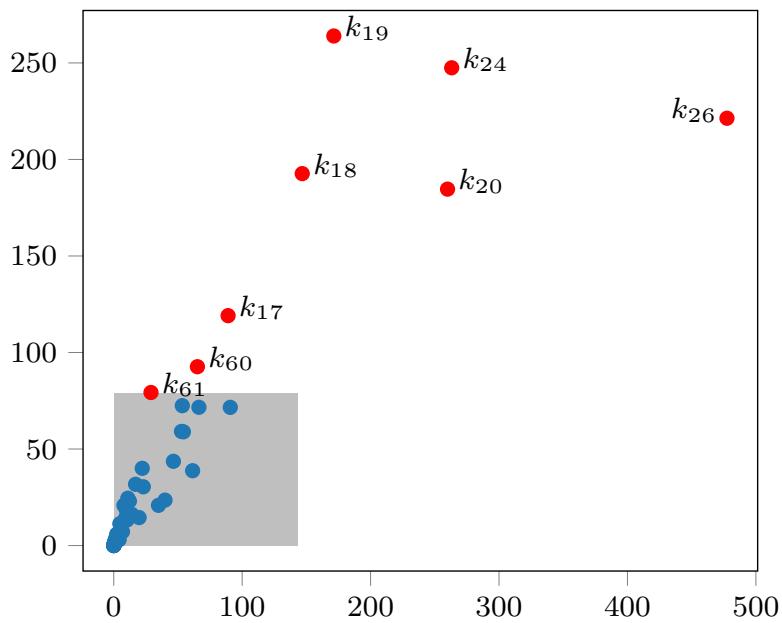

d)

$m_{2}$

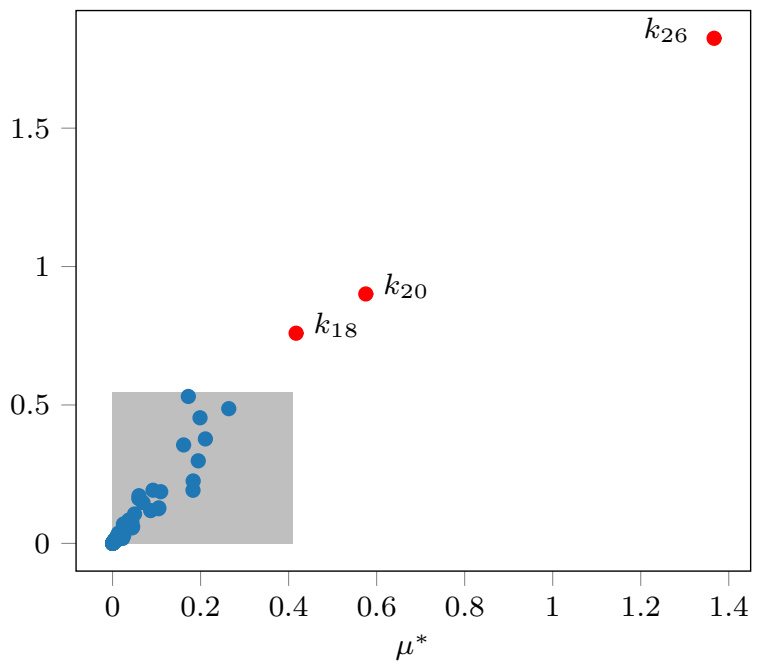

f)

Parameter impact

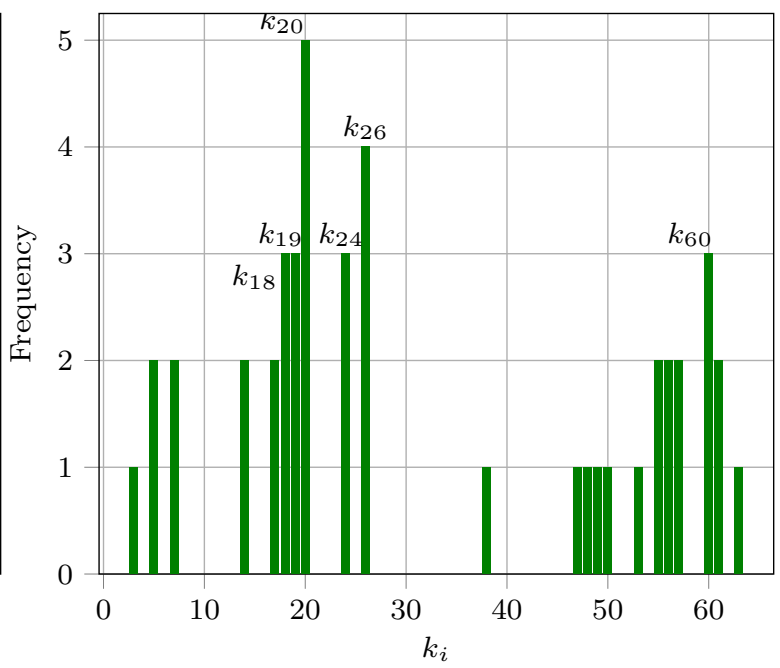

Fig. 5 Each QoI statistics ( $\sigma_{j}$ vs $\mu_{j}^{*}$ ) are evaluated through the Morris analysis. The rectangle represents the threshold retention zone, the parameter with effects larger than $0.3 \times \max \left(\sigma_{j}\right)$ and $0.3 \times \max \left(\mu_{j}^{*}\right)$ are considered relevant. A histogram showing how many QoI are sensitive to each parameter is also shown (bottom right). 
While those indices may be evaluated with MonteCarlo sampling type method, they are here approximated from a surrogate polynomial approximation of each QoI. These polynomial approximation were built from level-3 Stroud quadrature sampling via numerical projections on Legendre first-order orthogonal polynomials of $\boldsymbol{k}$ (Xiu 2010). In this case, only 126 simulations were performed. Figure 6 shows the Sobol' coefficients of the 63 parameters for $t_{l a g}$. The parameters marked with a cross are the parameters with large variance. In fact, a remarkable consistency with the results from the Morris method is observed for all the $Q o I$ (results for the 4 remaining $Q o I$ are not shown).

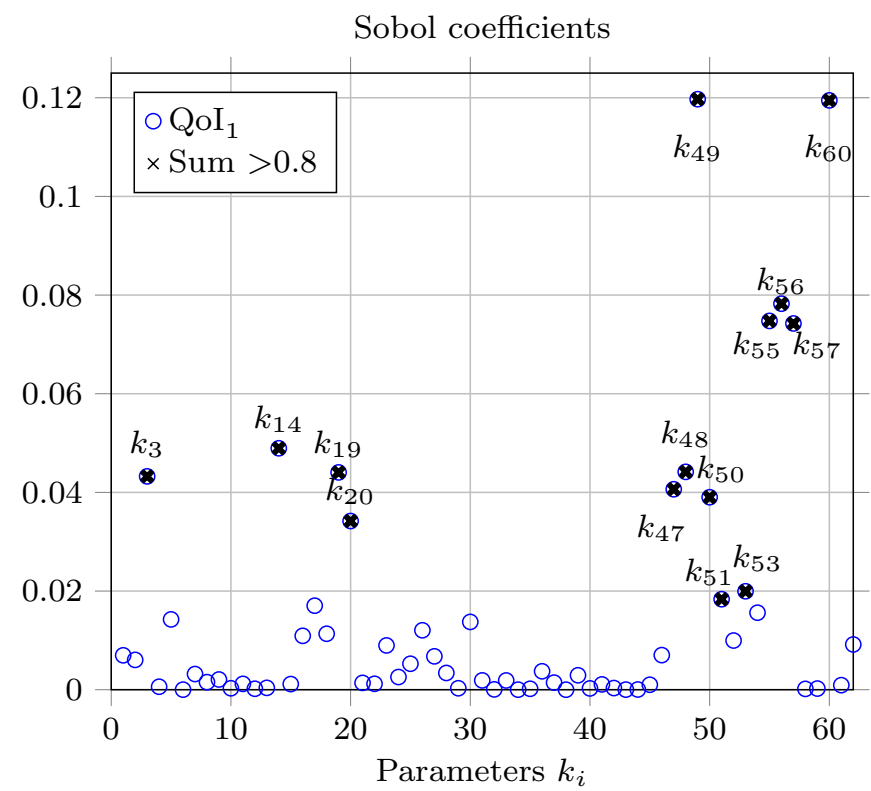

Fig. 6 Sobol' coefficients of the 63 parameters for $t_{l a g}$ calculated with 126 Stroud data points, with Legendre Polynomials. Coefficients marked with $\mathbf{x}$ are responsible for $80 \%$ of the output variance

\subsection{Reduced model for thrombin generation}

Whatever the confidence we have in a coagulation model, it can only be used in CFD if its computational cost remains tractable. As a result, model reduction is particularly useful to build coagulation models with a limited number of species. Of course, such models involve new parameters whose values need to be determined using reference data sets.

Eight thrombin generation assays initiated by contact activation were conducted on the same PNP sample to obtain the data sets relevant to thrombin. The mean of these thrombin formation curves was used to perform the Bayesian inference assuming a $4 \%$ of observation uncertainty. Optimal parameters obtained after 20, 000 MCMC (Metropolis-Hastings sampling algorithm) iterations are listed in Table 4.

\begin{tabular}{|c|c|c|}
\hline$\#$ & $\boldsymbol{k}$ & optimal \\
\hline 1 & $k_{1}$ & $6.4 \times 10^{-3}$ \\
2 & $k_{\text {cat }_{2}}$ & 8.21 \\
3 & $k_{m_{2}}$ & $8.95 \times 10^{-9}$ \\
4 & $k_{\text {cat }_{3}}$ & $3.5 \times 10^{-3}$ \\
5 & $k_{m_{3}}$ & $2.0 \times 10^{-9}$ \\
6 & $k_{\text {cat }_{4}}$ & 4.98 \\
7 & $k_{m_{4}}$ & $8.25 \times 10^{-7}$ \\
8 & $k_{5}$ & $7.79 \times 10^{3}$ \\
9 & $C_{X I I}$ & $9.36 \times 10^{-11}$ \\
11 & $C_{V}$ & $6.2 \times 10^{-9}$ \\
12 & $C_{A T I I I}$ & $1.665 \times 10^{-6}$ \\
\hline
\end{tabular}

Table 4 Optimal parameters obtained from Bayesian inference; (here taken as the statistical mode values) the initial concentration of prothrombin $C_{I I}=933.0 \mathrm{nM}$ is not infered.

Figure 7 shows the experimental data and the numerical results using the Bayesian optimal parameters in the reduced coagulation model. Numerical results using the Bayesian optimal parameters aligned well with TGA experimental data.

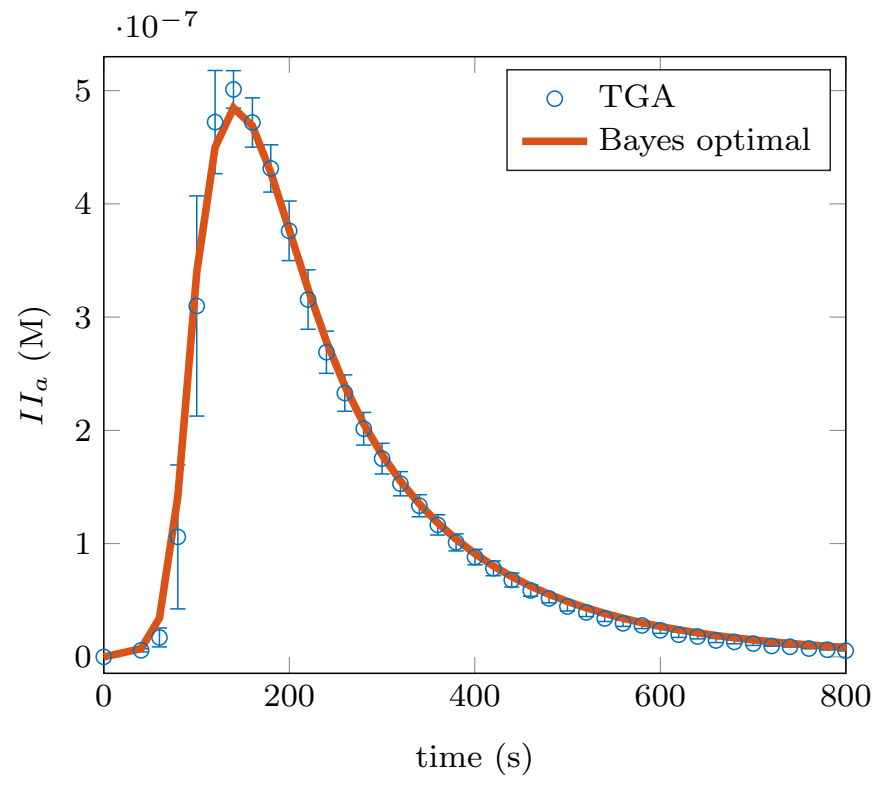

Fig. 7 Mean of the eight TGA data sets for physiological data and numerical thrombin formation using the reduced model (Fig. 4) with optimal parameters from Bayesian inference. The error bars represent the variance of the TGA data sets. The optimal parameters were obtained after 20000 MCMC iterations. 
To evaluate the predictive capabilities of the proposed reduced model, the initial condition of factor $I I$ was varied. Figure 8 shows both the numerical and TGA results for three prothrombin concentrations. The numerical results reproduce fairly well the trends of thrombin formation, demonstrating the good predictive capabilities of the reduced model using the Bayesian optimal parameters. The three cases correspond to the physiological condition $C_{I I}=933.0 \mathrm{nM}, 50 \%$ and $15 \%$ of the physiological values, respectively. It can be noticed that in both cases at low prothrombin concentrations are shifted with longer lag times than the TGA data. In addition, the amplitude of the $I I_{a}$ curves is, in both cases, smaller than the experimental results.

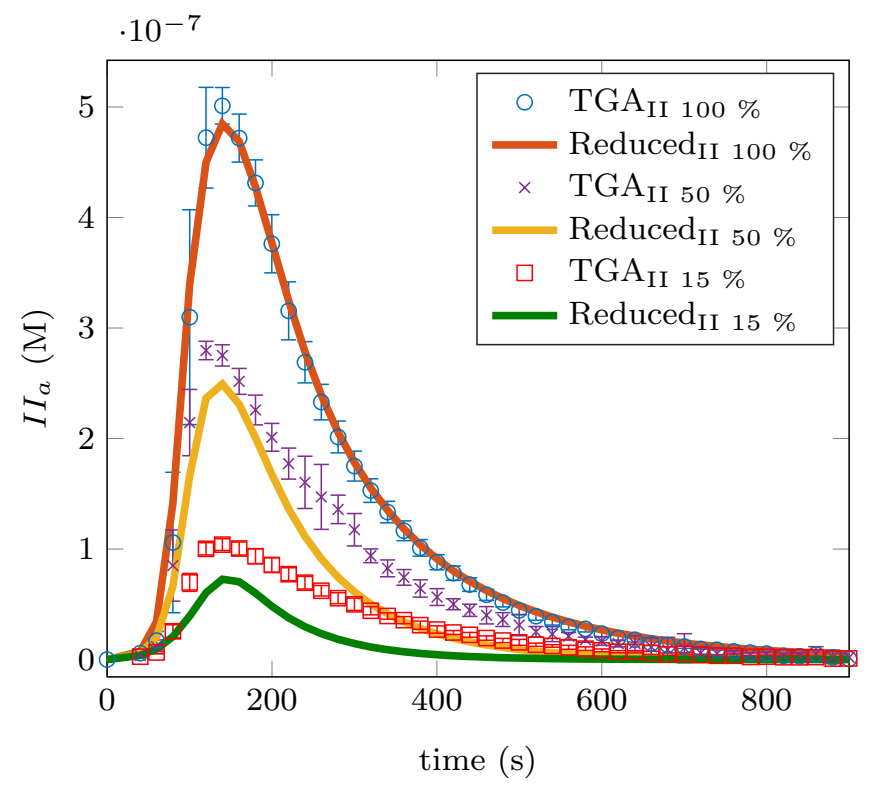

Fig. 8 Experimental TGA and numerical data using the reduced model with optimal parameters of Table 4 for a range of factor II initial concentrations, $100 \%=$ physiological PPP concentration, $50 \%, 15 \%$ )

The inference process was also repeated with a different set of reference data using the numerical data from the detailed model of Chatterjee et al. (2010) assuming an uncertainty on thrombin concentration of 4 $\%$. Table 5 shows the values of the inferred parameters. This approach can be applied to more general cases in which the experimental conditions differ from the TGA samples previously presented.

Figure 9 shows the thrombin generation curves using the inferred parameters of Table 5. A lag time between the model of Chatterjee et al. and the reduced model is observed for the case of $15 \%$ prothrombin ini-

\begin{tabular}{|c|c|c|}
\hline$\#$ & $\boldsymbol{k}$ & optimal \\
\hline 1 & $k_{1}$ & $1.2 \times 10^{-3}$ \\
2 & $k_{\text {cat }_{2}}$ & 0.28 \\
3 & $k_{m_{2}}$ & $3.72 \times 10^{-7}$ \\
4 & $k_{\text {cat }_{3}}$ & $7.7 \times 10^{-3}$ \\
5 & $k_{m_{3}}$ & $6.4 \times 10^{-10}$ \\
6 & $k_{\text {cat }_{4}}$ & 7.01 \\
7 & $k_{m_{4}}$ & $9.54 \times 10^{-7}$ \\
8 & $k_{5}$ & $9.88 \times 10^{3}$ \\
9 & $C_{X I I}$ & $7.91 \times 10^{-11}$ \\
11 & $C_{V}$ & $1.8 \times 10^{-8}$ \\
12 & $C_{A T I I I}$ & $1.977 \times 10^{-6}$ \\
\hline
\end{tabular}

Table 5 Optimal parameters obtained from Bayesian inference using the numerical data of the detailed model of Chatterjee et al. (2010). The initial concentration of prothrombin $C_{I I}=933.0 \mathrm{nM}$ is not infered.

tial concentration. A good trend is however observed in the reference and $50 \%$ cases.

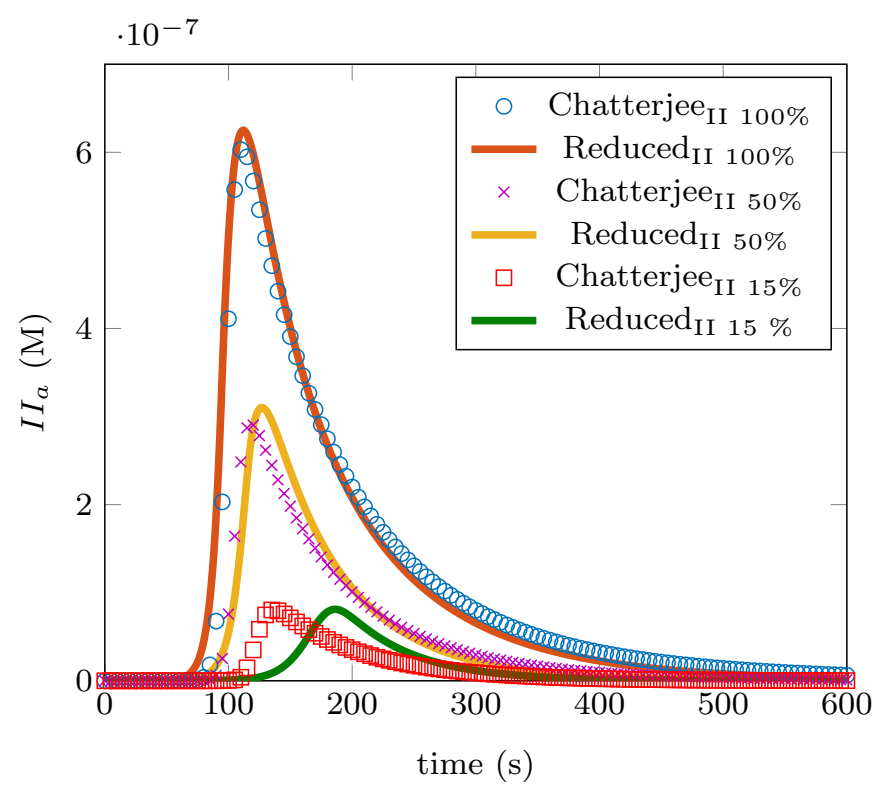

Fig. 9 Numerical results of the detailed model of Chatterjee et al. (2010) and numerical data using the reduced model with optimal parameters of Table 5 for a range of factor $I I$ initial concentrations, $100 \%=$ physiological PPP concentration, $50 \%, 15 \%$ )

\section{Discussion}

In this work, two kinetic models of the coagulation cascade initiated by contact activation with a different level of complexity are used: a full reference model from the literature and a reduced model introduced here for the first time. First, the Morris (1991) method for sensitivity analysis is applied to the existing detailed model of the coagulation cascade initiated by the contact ac- 
tivation system. This screening technique allowed the identification of the parameters that have a significant impact on the model QoI's. The kinetic rates that had the largest impact on the time before the reactions take up $t_{\text {lag }}$ are involved in the intrinsic coagulation pathway $\left(k_{57}, k_{55}, k_{60}, k_{56}, k_{48}, k_{49}\right)$. Interestingly, the reaction rate $k_{30}$ which is implicated in the contact activation of factor $X I I$ does not have a significant effect on $t_{\text {lag }}$, thus suggesting that its role is only to trigger the cascade of reactions. This results are consistent with the numerical results of Méndez Rojano et al. (2018) in which a parametric study on the activation rate of factor $X I I_{a}$ showed a limited impact on thrombin formation. This effect may explain the difficulties of developing a full non-thrombotic material since reducing the contact activation rate does not help to reduce the thrombin concentrations levels.

The ascending slope $m_{1}$ is particularly sensitive to $k_{50}$ which is involved in the auto-activation of factor $X I$. In addition parameters $k_{61}$ and $k_{63}$ participating in the formation of thrombin and activation of factor VIII showed large impact on this QoI. For the other quantities $\max \left(I I_{a}\right), m_{2}$ and ETP, the reaction rates that were identified correspond to reactions that are directly involved in the production of thrombin or the inhibition activity of $A T I I I\left(k_{26}, k_{20}, k_{23}, k_{24}, k_{18}\right.$, $\left.k_{19}\right)$.

To verify the results, another sensitivity analysis relying in the Stroud sampling quadrature and the Sobol' indices was performed and the results were qualitatively confirmed for all the QoI's. In the histogram showed in Fig. 5, parameter $k_{20}$ was identified to be important for all the QoI. Reaction rate $k_{26}$ is sensitive to four QoI except for $t_{\text {lag }}$. These findings align well with the sensitivity study of Danforth et al. (2009) on the coagulation model triggered by $T F=V I I_{a}$. Danforth and coworkers found that the thrombin formation levels were sensitive to variations on reaction rates $k_{26}$ and $k_{20}$. Many reaction rates identified by Danforth participate in the initiation phase of the extrinsic pathway. In the current work reactions rates $k_{57}, k_{56}$ and $k_{55}$ participating in the intrinsic system have a considerable impact on the QoI's $t_{l a g}$ and $m_{1}$. Finally, six reactions rates were found to be important to three output variables, $k_{18}, k_{19}, k_{24}$ which are involved in the production and inhibition of $m I I_{a}$ and $k_{60}$ which participates in the activation of factor $X$ by $I X_{a}$. A better experimental characterization of these six reactions may lead to an improvement in the robustness of the model. We emphasize that other uncertainty quantification techniques more accurate than Morris method also exist for a moderate number of random parameters (?). However, the parameters identified in the current work can be used to perform a more accurate sensitivity analysis on the most important parameters selected thanks to the Morris method, as done for instance by Link et al. (2018).

As pointed out by Ngoepe et al. (2018) thrombosis models should be sophisticated enough to provide precious clinical information with a reasonable computational cost. To tackle this challenge in the context of deviced-associated thrombosis, a reduced model for thrombin generation triggered by contact activation of factor $X I I$ is proposed. This model allows for the characterization of the patient thrombin formation profile with a minimal reaction mechanisms. The reduced model was constructed based on previous reduction approaches (Wagenvoord et al. 2006) and by substituting the extrinsic pathway with the contact activation system. In fact, the sensitivity results also guided the construction of the reduced model. For instance, the sensitivity analysis showed that formation of thrombin and meizothrombin by factor $V_{a}$ had an important role by means of parameters $k_{18}, k_{19}$ and $k_{20}$. In addition, inhibition activity of ATIII showed to be relevant through parameters $k_{26}$ and $k_{24}$. Thus, these two mechanisms were incorporated with the minimum number of reactions in the reduced model. Finally, in order to compute thrombin evolution, the 11 parameters involved in the model (kinetic rates and initial constants) have to be calibrated. A Bayesian inference framework with a MCMC sampling method was used to infer the optimal parameters using the concentration of thrombin in time as reference data from both the TGA and the detailed model of Chatterjee et al. (2010). The set of parameters found by the Bayesian inference method could reproduce the experimental TGA trend when the initial concentration of factor $I I$ was decreased to 50 and $15 \%$ from its physiological value. A supplemental set of parameter values was inferred using the detailed kinetic model of Chatterjee et al. The set of parameters aligned well with the trend presented by Chatterjee's model; this set of parameters may be used in general purpose CFD computations in which the conditions of the case are not the same as in our experimental configuration. Both sets of optimized parameters showed to follow a good trend with respect to TGA and numerical data showing equivalent predictive capabilities. Using such a reduced kinetic model in hemodynamic computations lowers the computational cost of chemical species transport by almost a factor of seven. 
The results contribute to improve the predicting capabilities of the model of Chatterjee et al. (2010). To the best of our knowledge, this is the first time that a minimal reaction mechanism triggered by the contact activation system is proposed. The reduced model combined with proper boundary conditions (Méndez Rojano et al. 2018) may be particularly useful in situations in which the coagulation reactions are triggered by contact activation of factor XII.

Acknowledgements We are grateful to the CONACyT, Mexico scholarship and the LabEx Numev (convention ANR-10LABX-20) for their financial support. This work was performed using HPC resources from GENCI-CINES (Grants 2016-c2015037194 and 2018-A0040307194) and with the support of the High Performance Computing Platform HPC@LR.

Conflict of Interest: The authors declare that they have no conflict of interest.

\section{References}

Alderazi YJ, Shastri D, Kass-Hout T, Prestigiacomo CJ, Gandhi CD (2014) Flow diverters for intracranial aneurysms. Stroke Res. and Treat.2014(415653):1-12

Alemu Y, Bluestein D (2007) Flow-induced platelet activation and damage accumulation in a mechanical heart valve: Numerical studies. Artif. Organs31(9):677-688, DOI https://doi.org/10.1111/j.1525-1594.2007.00446.x

Anand M, Rajagopal K, Rajagopal KR (2008) A model for the formation, growth, and lysis of clots in quiescent plasma. a comparison between the effects of antithrombin iii deficiency and protein c deficiency. J. Theor. Biol.253:725-738

Andrieu C, De Freitas N, Doucet A, Jordan MI (2003) An introduction to MCMC for machine learning. Mach. Learn.50:5-43

Ashyraliyev M, Fomekong-Nanfack Y, Kaandorp JA, Blom JG (2009) Systems biology: parameter estimation for biochemical models. FEBS J.276(4):886-902

Belyaev AV, Dunster J, Gibbins J, Panteleev M, Volpert VPoLR (2018) Modeling thrombosis in silico: Frontiers, challenges, unresolved problems and milestones. Phys. Life Rev.in press

Bhogal P, Martinez Moreno R, Ganslandt O, Bäzner H, Henkes H, Perez MA (2017) Use of flow diverters in the treatment of unruptured saccular aneurysms of the anterior cerebral artery. J. Neurointerv. Surg.9(3):283-289

Birolleau A, Potte G, Lucor D (2014) Adaptive bayesian inference for discontinuous inverse problems, application to hyperbolic conservation laws. Commun. Comput. Phys.16(1):1-34

Campolongo F, Cariboni J, Saltelli A (2007) An effective screening design for sensitivity analysis of large models. Environ. Mod. Soft.22(10):1509-1518, DOI https://doi.org/10.1016/j.envsoft.2006.10.004

Chatterjee MS, Denney WS, Jing H, Diamond SL (2010) Systems biology of coagulation initiation: Kinetics of thrombin generation in resting and activated human blood. PLoS Comp. Biol.6(9)
Chnafa C, Mendez S, Nicoud F (2016) Image-based simulations show important flow fluctuations in a normal left ventricle: What could be the implications? Ann. of Biomed. Eng.44(11):3346-3358

Danforth CM, Orfeo T, Mann KG, Brummel-Ziedins KE, Everse SJ (2009) The impact of uncertainty in a blood coagulation model. Math. Med. Biol.26(4):323-336, DOI https://doi.org/10.1093/imammb/dqp011

Danforth CM, Orfeo T, Everse SJ, Mann KG, BrummelZiedins KE (2012) Defining the boundaries of normal thrombin generation: Investigations into hemostasis. PLoS One7:1-12

De Biasi AR, Manning KB, Salemi A (2015) Science for surgeons: Understanding pump thrombogenesis in continuous-flow left ventricular assist devices. J. Thorac. Cardiovasc. Surg.149(3):667-673, DOI https://doi.org/10.1016/j.jtcvs.2014.11.041

Dumont K, Vierendeels J, Kaminsky R, van Nooten G, Verdonck P, Bluestein Dd (2007) Comparison of the hemodynamic and thrombogenic performance of two bileaflet mechanical heart valves using a CFD/FSI model. J. Biomech. Eng.129(4):558-565

Fogelson AL, Neeves KB (2015) Fluid mechanics of blood clot formation. Ann. Rev. Fluid Mech.47(1):377-403

Fogelson AL LK Hussain YH (2012) Blood clot formation under flow: The importance of factor xi depends strongly on platelet count. Biophys. J.102(1):10-18

Gorbet MB, Sefton MV (2004) Biomaterial-associated thrombosis: roles of coagulation factors, complement, platelets and leukocytes. Biomaterials25:5681-5703

Hastings SM, Ku DN, Wagoner S, Maher OK, Deshpande S (2017) Sources of circuit thrombosis in pediatric extracorporeal membrane oxygenation. Am. Soc. Artif. Intern. Org. 63(1):86-92

Hemker H, Giesen P, Al Dieri R, Regnault V, De Smedt E, Wagenvoord R, Lecompte T, Bguin S (2003) Calibrated automated thrombin generation measurement in clotting plasma. Pathophysiol. Haemost. Thromb.33:4-15

Hemker HC, Kerdelo S, Kremers RMW (2012) Is there value in kinetic modeling of thrombin generation? no (unless...). J. Thromb. Haemost.10:1470-1477

Hockin MF, Jones KC, Everse SJ, Mann KG (2002) A model for the stoichiometric regulation of blood coagulation. J. Biol. Chem.277(21):18322-18333

Indolfi C, De Rosa S, Colombo A (2016) Bioresorbable vascular scaffolds - basic concepts and clinical outcome. Nat. Rev. Cardiol.13:719-729

Iooss B, Lemaître P (2015) Uncertainty Management in Simulation-Optimization of Complex Systems: Algorithms and Applications, Springer US, chap A Review on Global Sensitivity Analysis Methods, pp 101-122

Jaffer IH, Fredenburgh JC, Hirsh J, Weitz JI (2015) Medical device-induced thrombosis: what causes it and how can we prevent it? J. Thromb. Haemost.13(Suppl. 1):72-81

Jones KC, Mann KG (1994) A model for the tissue factor pathway to thrombin. ii. a mathematical simulation. J. Biol. Chem.269:23367-23373

Kirklin JK, Pagani FD, Kormos RL, Stevenson LW, Blume ED, Myers SL, Miller MA, Baldwin JT, Young JB, Naftel DC (2017) Eighth annual intermacs report: Special focus on framing the impact of adverse events. J. Heart Long Transplant.36(10):1080-1086

Komiyama Y, Pedersen AH, Kisiel W (1990) Proteolytic activation of human factors ix and $\mathrm{x}$ by recombinant human factor viia: effects of calcium, phospholipids, and tissue factor. Biochemistry-US29:94189425 
Lanotte L, Mauer J, Mendez S, Fedosov DA, Fromental JM, Clavería V, Nicoud F, Gompper G, Abkarian M (2016) Red cells' dynamic morphologies govern blood shear thinning under microcirculatory flow conditions. Proc. Natl Acad. Sc. USA113(47):13289-13294, DOI 10.1073/pnas.1608074113

Leiderman K, Fogelson AL (2011) Grow with the flow: A spatial-temporal model of platelet deposition and blood coagulation under flow. Math. Med. Biol.28:47-84

Link KG, Stobb MT, Di Paola J, Neeves KB, Fogelson AL, Sindi SS, Leiderman K (2018) A local and global sensitivity analysis of a mathematical model of coagulation and platelet deposition under flow. PLoS One13(7):1-38

Mehra MR, Stewart GC, Uber PA (2014) The vexing problem of thrombosis in long-term mechanical circulatory support. J. Heart Long Transplant.33:1-11

Méndez Rojano R, Mendez S, Nicoud F (2018) Introducing the pro-coagulant contact system in the numerical assessment of device-related thrombosis. Biomech. Model. Mechanobiol.17(3):815-826

Morris M (1991) Factorial sampling plans for preliminary computational experiments. Technometrics33:161174

Naidu P, Anand M (2014) Importance of viiia inactivation in a mathematical model for the formation, growth, and lysis of clots. Math. Model. Nat. Phenom.9(6):17-33

Ngoepe MN, Ventikos Y (2016) Computational modelling of clot development in patient-specific cerebral aneurysm cases. J. Thromb. Haemost.14(2):262-272

Ngoepe MN, Frangi AF, Byrne JV, Ventikos Y (2018) Thrombosis in cerebral aneurysms and the computational modeling thereof: A review. Front. Physiol.9:306

Papadopoulos KP, Gavaises M, Atkin C (2014) A simplified mathematical model for thrombin generation. Med. Eng. Phys.36(2):196-204, DOI https://doi.org/10.1016/j.medengphy.2013.10.012

Saltelli A, Ratto M, Tarantola S, Campolongo F (2004) Sensitivity analysis practice: A guide to scientific models. John Wiley \& Sons, Ltd

Shadden SC, Hendabadi S (2013) Potential fluid mechanic pathways of platelet activation. Biomech. Model. Mechanobiol.12:467-474

Sigüenza J, Mendez S, Nicoud F (2017) How should the optical tweezers experiment be used to characterize the red blood cell membrane mechanics? Biomech. Model. Mechanobiol.16:1645-1657

Sigüenza J, Pott D, Mendez S, Sonntag S, Kaufmann TAS, Steinseifer U, Nicoud F (2018) Fluid-structure interaction of a pulsatile flow with an aortic valve model: A combined experimental and numerical study. Int. J. Numer. Meth. Biomed. Eng.34(e2945):1-19

Sobol IM (2001) Global sensitivity indices for rather complex mathematical models can be efficiently computed by Monte Carlo (or quasi-Monte Carlo) methods. these indices are used for estimating the influence of individual variables or groups of variables on the model output. / mcs 55:271-280

Sun JCJ, Davidson MJ, Lamy A, Eikelboom JW (2009) Antithrombotic management of patients with prosthetic heart valves: current evidence and future. The Lancet374(9689):565-576

Taylor JO, Meyer RS, Deutsch S, Manning KB (2016) Development of a computational model for macroscopic predictions of device-induced thrombosis. Biomech. Model. Mechanobiol.15(6):1713-1731

Wagenvoord R, Hemker PW, Hemker HC (2006) The limits of simulation of the clotting system. J. Thromb.
Haemost.4:1331-1338

Wilson WM, Cruden NL (2013) Advances in coronary stent technology: current expectations and new developments. Res. Rep. Clin. Cardio.4:85-96

Wu WT, Yang F, Wu J, Aubry N, Massoudi M, Antaki JF (2016) High fidelity computational simulation of thrombus formation in Thoratec Heart Mate II continuous flow ventricular assist device. Sc. Rep.6:38025-1-11, DOI $10.1038 /$ srep38025

Xiu D (2010) Numerical Methods for Stochastic Computations. Princeton University Press

Yan Y, Xu LC, Vogler EA, Siedlecki CA (2018) 1 - Contact activation by the intrinsic pathway of blood plasma coagulation. Woodhead Publishing

Yazdani A, Li H, Humphrey JD, Karniadakis GE (2017) A general shear-dependent model for thrombus formation. PLoS Comp. Biol.13(1):e1005291

Yoganathan AP, He Z, Jones SC (2004) Fluid mechanics of heart valves. Ann. Rev. Biomed. Eng.6:331-62

Zarnitsina VI, Pokhilko AV, Ataullakhanov FI (1996) A mathematical model for the spatio-temporal dynamics of intrinsic pathway of blood coagulation. i. the model description. Thromb. Res.84(4):225-236

Zhu D (2007) Mathematical modeling of blood coagulation cascade: kinetics of intrinsic and extrinsic pathways in normal and deficient conditions. Blood. Coagul. Fibribolysis.18:637-646

Zmijanovic V, Mendez S, Moureau V, Nicoud F (2017) About the numerical robustness of biomedical benchmark cases: Interlaboratory FDA's idealized medical device. Int. J. Numer. Meth. Biomed. Eng.33(1):e02789:1-17 